\title{
Moment Properties of Generalized Order Statistics from Exponential-Weibull Lifetime Distribution
}

\author{
R.U. Khan* and M.A. Khan \\ Department of Statistics and Operations Research, Aligarh Muslim University, Aligarh-202002, India \\ Email: aruke@rediffmail.com
}

\begin{abstract}
In this paper, simple explicit expressions and some recurrence relations satisfied by single and product moments of generalized order statistics from exponential-Weibull lifetime distribution have been obtained. These relations are deduced for moments of order statistics and upper record values. Further, conditional moments and a recurrence relation for single moments of the generalized order statistics are used to characterize this distribution and some computational works are also carried out.
\end{abstract}

Keywords: Exponential-Weibull lifetime distribution, generalized order statistics, record values, order statistics, single moments, product moments, conditional moments, recurrence relations, characterization.

\section{$1 \quad$ Introduction}

The concept of generalized order statistics (gos) was introduced by Kamps [1]. A variety of order models of random variables is contained in this concept, such as order statistics, upper record values, progressive Type II censoring order statistics, sequential order statistics and Pfeifer's records.

Let $X_{1}, X_{2}, \ldots$ be a sequence of independent and identically distributed (iid) random variables (rv) with distribution function $(d f) F(x)$ and probability density function $(p d f) f(x)$. Let $k>0, n \in N$, $m \in \mathfrak{R}$ and $\gamma_{r}=k+(n-r)(m+1)>0$. If the random variables $X(r, n, m, k), r=1,2, \ldots, n$ possess a joint $p d f$ of the form

$$
k\left(\prod_{j=1}^{n-1} \gamma_{j}\right)\left(\prod_{i=1}^{n-1}\left[1-F\left(x_{i}\right)\right]^{m} f\left(x_{i}\right)\right)\left[1-F\left(x_{n}\right)\right]^{k-1} f\left(x_{n}\right)
$$

on the cone $F^{-1}(0)<x_{1} \leq \ldots \leq x_{n}<F^{-1}(1)$, then they are called gos of a sample from a distribution with $d f F(x)$. Note that in the case $m=0, k=1$, this model reduces to the joint $p d f$ of the ordinary order statistics and when $m=-1$, we get the joint $p d f$ of the $k-$ th upper record values. In view of (1), the marginal $p d f$ of the $r$-th gos $X(r, n, m, k)$ is given by

$$
f_{X(r, n, m, k)}=\frac{C_{r-1}}{(r-1) !}[\bar{F}(x)]^{\gamma_{r}-1} f(x) g_{m}^{r-1}(F(x))
$$

and the joint $p d f$ of $X(r, n, m, k)$ and $X(s, n, m, k), 1 \leq r<s \leq n$, is

$$
f_{X(r, n, m, k), X(s, n, m, k)}=\frac{C_{s-1}}{(r-1) !(s-r-1) !}[\bar{F}(x)]^{m} f(x) g_{m}^{r-1}(F(x)) \times\left[h_{m}(F(y))-h_{m}(F(x))\right]^{s-r-1}[\bar{F}(y)]^{\gamma_{s}-1} f(y)
$$

where

$$
\begin{aligned}
& \bar{F}(x)=1-F(x), \quad C_{r-1}=\prod_{i=1}^{r} \gamma_{i}, \quad r=1,2, \ldots, n, \\
& h_{m}(x)= \begin{cases}-\frac{1}{m+1}(1-x)^{m+1}, & m \neq-1 \\
-\ln (1-x), & m=-1\end{cases}
\end{aligned}
$$


and $g_{m}(x)=h_{m}(x)-h_{m}(0), x \in[0,1)$.

Several authors utilized the concept of gos in their studies. References may be made to Kamps and Gather [2], Keseling [3], Cramer and Kamps [4], Ahsanullah [5], Habibullah and Ahsanullah [6], Raqab [7], Kamps and Cramer [8], Ahmad and Fawzy [9], Beiniek and Syznal [10], Al-Hussaini and Ahmad [11], Cramer et al. [12], Khan and Alzaid [13], Jaheen [14], Khan et al. ([15],[16]), Khan and Zia [17] among others.

Kamps [18] investigated the importance of recurrence relations of order statistics in characterization. In this paper, we study the generalized order statistics from exponential-Weibull lifetime distribution and derive explicit expressions for single moments. We also establish some simple recurrence relationships for the single and the product moments. Further, various deductions and particular cases are discussed. At the end, the characterization results based on conditional expectation and recurrence relations are presented and some computational works are also carried out.

A random variable $X$ is said to have exponential-Weibull lifetime distribution (Cordeiro et al. [19]) if its $p d f$ is of the form

$$
f(x)=\left(\alpha+\beta \theta x^{\theta-1}\right) e^{-\left(\alpha x+\beta x^{\theta}\right)}, x>0, \alpha>0, \beta>0, \theta>0
$$

and the corresponding $d f$ is

$$
F(x)=1-e^{-\left(\alpha x+\beta x^{\theta}\right)}, x>0, \alpha>0, \beta>0, \theta>0
$$

It can be seen that

$$
f(x)=\left(\alpha+\beta \theta x^{\theta-1}\right) \bar{F}(x)
$$

We can obtain several special models from relation (4). The exponential and Weibull distributions are the special cases for $\theta=1$ or $\theta=1, \alpha=0$ or $\beta=0$ and $\alpha=0$, respectively. The Rayleigh distribution arises when $\alpha=0$ and $\theta=2$. The two-parameter linear failure rate distribution is obtained when $\theta=2$.

The relation (6) will be used to derive explicit expressions and some recurrence relations for the moments of gos from exponential-Weibull lifetime distribution.

\section{Relations for Single Moments}

We shall first establish the existence of $E\left[X^{j}(r, n, m, k)\right]$. Using $(2)$, we have when $m \neq-1$

$$
E\left[X^{j}(r, n, m, k)\right]=\frac{C_{r-1}}{(r-1) !} \int_{0}^{\infty} x^{j}[\bar{F}(x)]^{\gamma_{r}-1} f(x) g_{m}^{r-1}(F(x)) \mathrm{d} x
$$

By using binomial expansion, (7) can be rewritten as

$$
E\left[X^{j}(r, n, m, k)\right]=\frac{C_{r-1}}{(r-1) !(m+1)^{r-1}} \sum_{u=0}^{r-1}(-1)^{u}\left(\begin{array}{c}
r-1 \\
u
\end{array}\right) \int_{0}^{\infty} x^{j}[\bar{F}(x)]^{\gamma_{r-u}-1} f(x) \mathrm{d} x
$$

Further, on using (6) in (8), we obtain

$$
\begin{aligned}
E\left[X^{j}(r, n, m, k)\right] & =A \int_{0}^{\infty} x^{j}[\bar{F}(x)]^{\gamma_{r-u}}\left(\alpha+\beta \theta x^{\theta-1}\right) \mathrm{d} x \\
& =-\frac{A}{\gamma_{r-u}} \int_{0}^{\infty} x^{j}\left(\frac{d\left(e^{-\gamma_{r-u}\left(\alpha x+\beta x^{\theta}\right)}\right)}{d x}\right) \mathrm{d} x
\end{aligned}
$$

where $A=\frac{C_{r-1}}{(r-1) !(m+1)^{r-1}} \sum_{u=0}^{r-1}(-1)^{u}\left(\begin{array}{c}r-1 \\ u\end{array}\right)$.

Integrating by parts now yields

On expanding $e^{-\gamma_{r-u} \alpha x}$ in Taylor series, we get

$$
E\left[X^{j}(r, n, m, k)\right]=\frac{j A}{\gamma_{r-u}} \int_{0}^{\infty} x^{j-1} e^{-\gamma_{r-u}\left(\alpha x+\beta x^{\theta}\right)} \mathrm{d} x
$$

$$
E\left[X^{j}(r, n, m, k)\right]=A^{*} \int_{0}^{\infty} x^{j+v-1} e^{-\gamma_{r-u} \beta x^{\theta}} \mathrm{d} x
$$


where $A^{*}=\frac{j A}{\gamma_{r-u}} \sum_{v=0}^{\infty}(-1)^{v} \frac{\left(\alpha \gamma_{r-u}\right)^{v}}{v !}$.

We have $[20]$

$$
\int_{0}^{\infty} x^{m} e^{-\beta x^{n}} d x=\frac{\Gamma(m+1) / n}{n \beta^{(m+1) / n}}, \beta, m, n>0
$$

Now on substituting (10) in (9), we have

$$
E\left[X^{j}(r, n, m, k)\right]=\frac{j C_{r-1}}{(r-1) ! \theta(m+1)^{r-1}} \sum_{v=0}^{\infty} \sum_{u=0}^{r-1}(-1)^{u+v}\left(\begin{array}{c}
r-1 \\
u
\end{array}\right) \times \frac{\alpha^{v}\left(\gamma_{r-u}\right)^{v-1-(j+v) / \theta}}{v ! \beta^{(j+v) / \theta}} \Gamma\{(j+v) / \theta\}
$$

When $m=-1$,

$$
E\left[X^{j}(r, n, m, k)\right]=\frac{0}{0} \text { as } \sum_{u=0}^{r-1}(-1)^{u}\left(\begin{array}{c}
r-1 \\
u
\end{array}\right)=0
$$

Since (11) is of the form $\frac{0}{0}$ at $m=-1$, therefore, we have

$$
E\left[X^{j}(r, n, m, k)\right]=B \sum_{u=0}^{r-1}(-1)^{u}\left(\begin{array}{c}
r-1 \\
u
\end{array}\right) \frac{[k+(n-r+u)(m+1)]^{v-\{(j+v) / \theta\}-1}}{(m+1)^{r-1}}
$$

where $B=\frac{j C_{r-1}}{(r-1) ! \theta} \sum_{v=0}^{\infty}(-1)^{v} \frac{\alpha^{v} \Gamma\{(j+v) / \theta\}}{v ! \beta^{(j+v) / \theta}}$.

Differentiating numerator and denominator of $(12)(r-1)$ times with respect to $m$, we get

$$
\begin{aligned}
E\left[X^{j}(r, n, m, k)\right]= & B \sum_{u=0}^{r-1}(-1)^{u+(r-1)}\left(\begin{array}{c}
r-1 \\
u
\end{array}\right) \\
& \times \frac{[\{(j+v) / \theta\}+1-v][\{(j+v) / \theta\}+2-v] \ldots[\{(j+v) / \theta\}+r-1-v](n-r+u)^{r-1}}{(r-1) ![k+(n-r+u)(m+1)]^{\{(j+v) / \theta\}+r-v}}
\end{aligned}
$$

On applying L' Hospital rule, we have

$$
\begin{aligned}
\lim _{m \rightarrow-1} E\left[X^{j}(r, n, m, k)\right]= & B \frac{[\{(j+v) / \theta\}+1-v] \ldots[\{(j+v) / \theta\}+r-1-v]}{(r-1) ! k^{\{(j+v) / \theta\}+r-v}} \\
& \times \sum_{u=0}^{r-1}(-1)^{u}\left(\begin{array}{c}
r-1 \\
u
\end{array}\right)(r-n-u)^{r-1}
\end{aligned}
$$

But for all integers $n \geq 0$ and for all real numbers $x$, we have [21]

$$
\sum_{i=0}^{n}(-1)^{i}\left(\begin{array}{l}
n \\
i
\end{array}\right)(x-i)^{n}=n !
$$

Substitute (14) in (13) and after simplification, we find that

$$
E\left[X^{j}(r, n,-1, k)\right]=E\left[\left(Y_{r}^{(k)}\right)^{j}\right]=\frac{j}{(r-1) ! \theta} \sum_{v=0}^{\infty}(-1)^{v} \frac{\alpha^{v} \Gamma\{(j+v) / \theta\} \Gamma\{((j+v) / \theta)-v+r\}}{v ! k^{\{(j+v) / \theta\}-v} \beta^{(j+v) / \theta} \Gamma\{((j+v) / \theta)-v+1\}}
$$

where $Y_{r}^{(k)}$ denotes the $k$-th upper record values.

Remark 1. Putting $\alpha=0$ in (11), we get the explicit expression for single moments of gos from the Weibull distribution as given by Kamps [1] pp-101.

Remark 2. Putting $\theta=1$ in (11), the results for single moments of gos from exponential distribution with parameter $(\alpha+\beta)$ is deduced in the form

$$
E\left[X^{j}(r, n, m, k)\right]=\frac{C_{r-1}}{(r-1) !(m+1)^{r-1}(\alpha+\beta)^{j}} \sum_{u=0}^{r-1}(-1)^{u}\left(\begin{array}{c}
r-1 \\
u
\end{array}\right) \frac{\Gamma(j+1)}{\left(\gamma_{r-u}\right)^{j+1}}
$$

Remark 3. Setting $\theta=1$ and $\alpha=0$ in (11), we get the explicit expression for single moments of gos from the exponential distribution, established by Kamps [1] pp-101.

Remark 4. Setting $\alpha=0$ in (15), the result for single moments of $k$-th upper record values is deduced for the Weibull distribution, which verifies the result obtained by Kamps [1] pp-101. 
Remark 5. At $\theta=1$ in (15), the result for single moments of $k$-th upper record values is deduced for the exponential distribution with parameter $(\alpha+\beta)$ in the form

$$
E\left[X^{j}(r, n,-1, k)\right]=E\left[\left(Y_{r}^{(k)}\right)^{j}\right]=\frac{\Gamma(j+r)}{(r-1) ! k^{j}(\alpha+\beta)^{j}}
$$

Remark 6. Setting $\theta=1$ and $\alpha=0$ in (15), the result for single moments of $k$-th upper record values is deduced for the exponential distribution as given by Kamps [1] pp-101.

\section{Special cases}

i) Putting $m=0$ and $k=1$ in (11), the exact expression for the single moments of order statistics from exponential-Weibull lifetime distribution can be obtained as

$$
E\left(X_{r: n}^{j}\right)=\frac{j C_{r: n}}{\theta} \sum_{v=0}^{\infty} \sum_{u=0}^{r-1}(-1)^{u+v}\left(\begin{array}{c}
r-1 \\
u
\end{array}\right) \frac{\alpha^{v}(n-r+u+1)^{v-1-(j+v) / \theta}}{v ! \beta^{(j+v) / \theta}} \Gamma\{(j+v) / \theta\}
$$

where $C_{r: n}=\frac{n !}{(r-1) !(n-r) !}$.

ii) Putting $k=1$ in (15), we deduce the explicit formula for the single moments of upper records for exponential-Weibull lifetime distribution in the form

$$
E\left[\left(Y_{r}^{(1)}\right)^{j}\right]=E\left[X_{U(r)}^{j}\right]=\frac{j}{(r-1) ! \theta} \sum_{v=0}^{\infty}(-1)^{v} \times \frac{\alpha^{v} \Gamma\{(j+v) / \theta\} \Gamma\{((j+v) / \theta)-v+r\}}{v ! \beta^{(j+v) / \theta} \Gamma\{((j+v) / \theta)-v+1\}}
$$

Expressions (16) and (17) can be used to obtain the moments of order statistics and upper record values for arbitrary chosen values of $\alpha, \beta, \theta$ and various sample size $n=1,2, \ldots, 5$. Some numerical computations for the first four moments of order statistics and upper record values from exponential-

\begin{tabular}{|c|c|c|c|c|c|c|c|c|c|}
\hline \multirow{3}{*}{$n$} & \multirow{3}{*}{$r$} & \multicolumn{4}{|c|}{$\alpha=1, \quad \theta=3$} & \multicolumn{4}{|c|}{$\alpha=1 \quad \theta=3$} \\
\hline & & \multicolumn{4}{|c|}{$\beta=1$} & \multicolumn{4}{|c|}{$\dot{\beta}=2$} \\
\hline & & $E(X)$ & $E\left(X^{2}\right)$ & $E\left(X^{3}\right)$ & $E\left(X^{4}\right)$ & $E(X)$ & $E\left(X^{2}\right)$ & $E\left(X^{3}\right)$ & $E\left(X^{4}\right)$ \\
\hline 1 & 1 & 0.4847 & 0.4257 & 0.4214 & 0.4564 & 0.4123 & 0.2975 & 0.2401 & 0.2111 \\
\hline \multirow{2}{*}{2} & 1 & 0.2524 & 0.1633 & 0.1207 & 0.0985 & 0.2285 & 0.1236 & 0.0756 & 0.0506 \\
\hline & 2 & 0.7169 & 0.6880 & 0.7221 & 0.8143 & 0.5960 & 0.4714 & 0.4047 & 0.3716 \\
\hline \multirow{3}{*}{3} & 1 & 0.1574 & 0.0835 & 0.0512 & 0.0349 & 0.1492 & 0.0672 & 0.0345 & 0.0195 \\
\hline & 2 & 0.4425 & 0.3229 & 0.2598 & 0.2257 & 0.3871 & 0.2365 & 0.1578 & 0.1129 \\
\hline & 3 & 0.8541 & 0.8706 & 0.9533 & 1.1086 & 0.7005 & 0.5888 & 0.5282 & 0.5009 \\
\hline \multirow{4}{*}{4} & 1 & 0.1074 & 0.0489 & 0.0260 & 0.0154 & 0.1056 & 0.0413 & 0.0186 & 0.0093 \\
\hline & 2 & 0.3074 & 0.1873 & 0.1268 & 0.0932 & 0.2802 & 0.1447 & 0.0822 & 0.0502 \\
\hline & 3 & 0.5775 & 0.4585 & 0.3928 & 0.3582 & 0.4939 & 0.3282 & 0.2334 & 0.1756 \\
\hline & 4 & 0.9463 & 1.0080 & 1.1401 & 1.3588 & 0.7693 & 0.6757 & 0.6265 & 0.6093 \\
\hline \multirow{5}{*}{5} & 1 & 0.0776 & 0.0311 & 0.0147 & 0.0078 & 0.0785 & 0.0274 & 0.0110 & 0.0049 \\
\hline & 2 & 0.2266 & 0.1200 & 0.0712 & 0.0460 & 0.2138 & 0.0971 & 0.0487 & 0.0264 \\
\hline & 3 & 0.4286 & 0.2882 & 0.2103 & 0.1640 & 0.3798 & 0.2163 & 0.1323 & 0.0860 \\
\hline & 4 & 0.6769 & 0.5721 & 0.5144 & 0.4877 & 0.5700 & 0.4028 & 0.3007 & 0.2354 \\
\hline & 5 & 1.0137 & 1.1169 & 1.2965 & 1.5766 & 0.8192 & 0.7439 & 0.7079 & 0.7028 \\
\hline \multirow{3}{*}{$n$} & \multirow{3}{*}{$r$} & & $\alpha=2$, & $\theta=3$ & & & $\alpha=2$ & $\theta=3$ & \\
\hline & & \multicolumn{4}{|c|}{$\beta=1$} & \multicolumn{4}{|c|}{$\beta=2$} \\
\hline & & $E(X)$ & $E\left(X^{2}\right)$ & $E\left(X^{3}\right)$ & $E\left(X^{4}\right)$ & $E(X)$ & $E\left(X^{2}\right)$ & $E\left(X^{3}\right)$ & $E\left(X^{4}\right)$ \\
\hline 1 & 1 & 0.2796 & 0.2131 & 0.1882 & 0.1851 & 0.2524 & 0.1633 & 0.1207 & 0.0985 \\
\hline \multirow{2}{*}{2} & 1 & 0.1094 & 0.0567 & 0.0350 & 0.0245 & 0.1074 & 0.0489 & 0.0260 & 0.0154 \\
\hline & 2 & 0.4497 & 0.3696 & 0.3415 & 0.3457 & 0.3974 & 0.2777 & 0.2155 & 0.1816 \\
\hline
\end{tabular}
Weibull lifetime distribution are given in Table 1, 2, respectively.

Table 1. First four moments of order statistics 


\begin{tabular}{c|c|cccc|cccc}
\hline \multirow{3}{*}{3} & 1 & 0.0568 & 0.0226 & 0.0109 & 0.0060 & 0.0584 & 0.0210 & 0.0089 & 0.0043 \\
& 2 & 0.2148 & 0.1250 & 0.0831 & 0.0613 & 0.2054 & 0.1048 & 0.0601 & 0.0377 \\
& 3 & 0.5671 & 0.4918 & 0.4706 & 0.4880 & 0.4934 & 0.3642 & 0.2932 & 0.2535 \\
\hline \multirow{4}{*}{4} & 1 & 0.0341 & 0.0110 & 0.0044 & 0.0020 & 0.0361 & 0.0107 & 0.0038 & 0.0015 \\
& 2 & 0.1248 & 0.0573 & 0.0305 & 0.0182 & 0.1254 & 0.0517 & 0.0242 & 0.0125 \\
& 3 & 0.3048 & 0.1927 & 0.1358 & 0.1044 & 0.2853 & 0.1578 & 0.0959 & 0.0630 \\
& 4 & 0.6545 & 0.5915 & 0.5823 & 0.6158 & 0.5628 & 0.4330 & 0.3589 & 0.3170 \\
\hline \multirow{4}{*}{5} & 1 & 0.0224 & 0.0066 & 0.0020 & 0.0008 & 0.0242 & 0.0061 & 0.0019 & 0.0006 \\
& 2 & 0.0806 & 0.0307 & 0.0137 & 0.0069 & 0.0837 & 0.0291 & 0.011 & 0.0052 \\
& 3 & 0.1912 & 0.0973 & 0.0558 & 0.0351 & 0.1880 & 0.2163 & 0.0431 & 0.0236 \\
& 4 & 0.3806 & 0.2563 & 0.1891 & 0.1506 & 0.3502 & 0.2061 & 0.1312 & 0.0892 \\
& 5 & 0.7230 & 0.6753 & 0.6806 & 0.7321 & 0.6160 & 0.4897 & 0.4158 & 0.7028 \\
\hline
\end{tabular}

We can note that the relation $\sum_{i=1}^{n} E\left(X_{i: n}^{j}\right)=n E\left(X^{j}\right)$ (David and Nagaraja [22]) is satisfied here.

Table 2. First four moments of upper record values

\begin{tabular}{|c|c|c|c|c|c|c|c|c|}
\hline \multirow{3}{*}{$n$} & & $\alpha=1$ & $\theta=3$ & & & $\alpha=1$ & $\theta=3$ & \\
\hline & \multicolumn{4}{|c|}{$\beta=1$} & \multicolumn{4}{|c|}{$\beta=2$} \\
\hline & $\overline{E(X)}$ & $E\left(X^{2}\right)$ & $E\left(X^{3}\right)$ & $E\left(X^{4}\right)$ & $E(X)$ & $E\left(X^{2}\right)$ & $E\left(X^{3}\right)$ & $E\left(X^{4}\right)$ \\
\hline 1 & 0.56889 & 0.45576 & 0.43111 & 0.45468 & 0.49141 & 0.32772 & 0.25429 & 0.21836 \\
\hline 2 & 0.91044 & 0.95121 & 1.08956 & 1.3383 & 0.76444 & 0.65921 & 0.61777 & 0.61749 \\
\hline 3 & 1.14476 & 1.41461 & 1.85524 & 2.55571 & 0.94710 & 0.95972 & 1.02645 & 1.14873 \\
\hline 4 & 1.32344 & 1.84239 & 2.67656 & 4.03511 & 1.0857 & 1.23354 & 1.45714 & 1.78128 \\
\hline 5 & 1.46938 & 2.24072 & 3.53063 & 5.72872 & 1.19903 & 1.48698 & 1.90049 & 2.49615 \\
\hline \multirow{3}{*}{$n$} & & $\alpha=2$, & $\theta=3$ & & & $\alpha=2$ & $\theta=3$ & \\
\hline & \multicolumn{4}{|c|}{$\beta=1$} & \multicolumn{4}{|c|}{$\beta=2$} \\
\hline & $E(X)$ & $E\left(X^{2}\right)$ & $E\left(X^{3}\right)$ & $E\left(X^{4}\right)$ & $E(X)$ & $E\left(X^{2}\right)$ & $E\left(X^{3}\right)$ & $E\left(X^{4}\right)$ \\
\hline 1 & 0.39750 & 0.25454 & 0.20498 & 0.19060 & 0.36227 & 0.20163 & 0.13772 & 0.10710 \\
\hline 2 & 0.69970 & 0.60646 & 0.60059 & 0.65433 & 0.61746 & 0.45846 & 0.38254 & 0.34751 \\
\hline 3 & 0.93362 & 0.98443 & 1.13275 & 1.39520 & 0.80598 & 0.72009 & 0.69402 & 0.71042 \\
\hline 4 & 1.12136 & 1.36087 & 1.75728 & 2.38843 & 0.95367 & 0.97240 & 1.04633 & 1.17799 \\
\hline 5 & 1.27750 & 1.72650 & 2.44491 & 3.60436 & 1.07519 & 1.21276 & 1.42480 & 1.73455 \\
\hline
\end{tabular}

Now, we obtain the recurrence relations for single moments of exponential-Weibull lifetime distribution in the following theorem.

Theorem 1. For the distribution as given in (5) and $n \in N, m \in \mathfrak{R}, 1 \leq r \leq n, j=0,1,2, \ldots$

$$
\begin{aligned}
E\left(X^{j}(r, n, m, k)\right)= & \frac{\alpha \gamma_{r}}{j+1}\left\{E\left[X^{j+1}(r, n, m, k)\right]-E\left[X^{j+1}(r-1, n, m, k)\right]\right\}+\frac{\beta \theta \gamma_{r}}{j+\theta} \\
& \times\left\{E\left[X^{j+\theta}(r, n, m, k)\right]-E\left[X^{j+\theta}(r-1, n, m, k)\right]\right\}
\end{aligned}
$$

Proof. From (2) and (6), we have

$$
E\left[X^{j}(r, n, m, k)\right]=\frac{C_{r-1}}{(r-1) !}\left\{\alpha \int_{0}^{\infty} x^{j}[\bar{F}(x)]^{\gamma_{r}} g_{m}^{r-1}(F(x)) \mathrm{d} x+\beta \theta \int_{0}^{\infty} x^{j+\theta-1}[\bar{F}(x)]^{\gamma_{r}} g_{m}^{r-1}(F(x)) \mathrm{d} x\right\}
$$

Now (18) can be seen by noting that in view of Athar and Islam [23]

$$
E\left[X^{j}(r, n, m, k)\right]-E\left[X^{j}(r-1, n, m, k)\right]=\frac{j C_{r-2}}{(r-1) !} \int_{0}^{\infty} x^{j-1}[\bar{F}(x)]^{\gamma_{r}} g_{m}^{r-1}(F(x)) \mathrm{d} x
$$

Remark 7. Substituting $m=0, k=1$ in (18), we deduce the recurrence relation for single moments of order statistics from exponential-Weibull lifetime distribution in the form

$$
E\left(X_{r: n}^{j}\right)=\frac{\alpha(n-r+1)}{j+1}\left\{E\left(X_{r: n}^{j+1}\right)-E\left(X_{r-1: n}^{j+1}\right)\right\}+\frac{\beta \theta(n-r+1)}{j+\theta}\left\{E\left(X_{r: n}^{j+\theta}\right)-E\left(X_{r-1: n}^{j+\theta}\right)\right\}
$$


At $\theta=1, \alpha=0$, the result for single moments of order statistics is deduced for exponential distribution as given in Kamps [1] pp-122.

Remark 8. Putting $m=-1$ in (18), the result for single moments obtained by Khan et al. [24] for upper $k$-th record values from exponential-Weibull lifetime distribution is deduced.

Remark 9. Setting $\alpha=0$ in (18), we get the recurrence relation for single moments of gos from the Weibull distribution, obtained by Khan et al. [25] for $j=j-\theta$.

Remark 10. Assuming $\beta=0$ and $\alpha=1$ in (18), the result for single moments of gos is deduced for exponential distribution, established by Pawlas and Syznal [26].

Remark 11. By putting $\theta=2$ in (18), the result for single moments of gos obtained by Ahmad [27] with $\beta=v / 2$ for linear failure rate distribution is deduced.

Remark 12. By putting $\alpha=0$ and $\theta=2$ in (18), the recurrence relation for single moments of gos is deduced for Rayleigh distribution in the form

$$
E\left(X^{j}(r, n, m, k)\right)=\frac{2 \beta \gamma_{r}}{j+2}\left\{E\left[X^{j+2}(r, n, m, k)\right]-E\left[X^{j+2}(r-1, n, m, k)\right]\right\}
$$

\section{$3 \quad$ Relations for Product Moments}

Theorem 2. For the given exponential-Weibull distribution in (5) and $n \in N, m \in \mathfrak{R}, 1 \leq r<s \leq n$ and $i, j \geq 0$,

$$
\begin{aligned}
E\left[X^{i}(r, n, m, k) X^{j}(s, n, m, k)\right]= & \frac{\alpha \gamma_{s}}{(j+1)}\left\{E\left[X^{i}(r, n, m, k) X^{j+1}(s, n, m, k)\right]-E\left[X^{i}(r, n, m, k) X^{j+1}(s-1, n, m, k)\right]\right\} \\
& +\frac{\beta \gamma_{s}}{(j+\theta)} \times\left\{E\left[X^{i}(r, n, m, k) X^{j+\theta}(s, n, m, k)\right]-E\left[X^{i}(r, n, m, k) X^{j+\theta}(s-1, n, m, k)\right\}\right.
\end{aligned}
$$

Proof. From (3) and (6), we have

$$
\begin{aligned}
E\left[X^{i}(r, n, m, k) X^{j}(s, n, m, k)\right]= & \frac{\alpha C_{s-1}}{(r-1) !(s-r-1) !} \int_{0}^{\infty} \int_{x}^{\infty} x^{i} y^{j}[\bar{F}(x)]^{m} f(x) g_{m}^{r-1}(F(x)) \\
& \times\left[h_{m}(F(y))-h_{m}(F(x))\right]^{s-r-1}[\bar{F}(y)]^{\gamma_{s}} \mathrm{~d} y \mathrm{~d} x \\
& +\frac{\beta \theta C_{s-1}}{(r-1) !(s-r-1) !} \int_{0}^{\infty} \int_{x}^{\infty} x^{i} y^{j+\theta-1} \\
& \times[\bar{F}(x)]^{m} f(x) g_{m}^{r-1}(F(x))\left[h_{m}(F(y))-h_{m}(F(x))\right]^{s-r-1}[\bar{F}(y)]^{\gamma_{s}} \mathrm{~d} y \mathrm{~d} x
\end{aligned}
$$

In view of Athar and Islam [23], note that

$$
\begin{aligned}
& E\left[X^{i}(r, n, m, k) X^{j}(s, n, m, k)\right]-E\left[X^{i}(r, n, m, k) X^{j}(s-1, n, m, k)\right]=\frac{j C_{s-2}}{(r-1) !(s-r-1) !} \\
& \times \int_{0}^{\infty} \int_{x}^{\infty} x^{i} y^{j-1}[\bar{F}(x)]^{m} f(x) g_{m}^{r-1}(F(x))\left[h_{m}(F(y))-h_{m}(F(x))\right]^{s-r-1}[\bar{F}(y)]^{\gamma_{s}} d y d x
\end{aligned}
$$

Substitute (22) in (21) and after simplification, we get the result given in (20).

Remark 13. At $i=0$ in (20), the recurrence relation for product moments reduces to relation for single moments as obtained in (18).

Remark 14. Putting $m=0$ and $k=1$ in (20), we obtain the recurrence relation for the product moments of order statistics from the exponential-Weibull lifetime distribution as

$$
E\left(X_{r: n}^{i} X_{s: n}^{j}\right)=\frac{\alpha(n-s+1)}{(j+1)}\left\{E\left(X_{r: n}^{i} X_{s: n}^{j+1}\right)-E\left(X_{r: n}^{i} X_{s-1: n}^{j+1}\right)\right\}+\frac{\beta(n-s+1)}{(j+\theta)} \times\left\{E\left(X_{r: n}^{i} X_{s: n}^{j+\theta}\right)-E\left(X_{r: n}^{i} X_{s-1: n}^{j+\theta}\right)\right\}
$$

At $\theta=1, \alpha=0$, the relation for product moments of order statistics is deduced for exponential distribution as

$$
E\left(X_{r: n}^{i} X_{s: n}^{j}\right)=\frac{\beta(n-s+1)}{(j+1)}\left\{E\left(X_{r: n}^{i} X_{s: n}^{j+1}\right)-E\left(X_{r: n}^{i} X_{s-1: n}^{j+1}\right)\right\}
$$

Remark 15. Setting $m=-1$ in Theorem 2, the relation for the product moments in Khan et al. [24] for upper $k$-th record values from the exponential-Weibull lifetime distribution is deduced. 
Remark 16. Setting $\alpha=0$ in (20), we get the recurrence relation for the product moments of gos from the Weibull distribution as obtained by Khan et al. [25] for $j=j-\theta$.

Remark 17. Assuming $\beta=0$ and $\alpha=1$ in (20), the result for the product moments of gos is deduced for the exponential distribution, established by Pawlas and Syznal [26].

Remark 18. By putting $\theta=2$ in (20), the result for product moments of gos obtained by Ahmad [27] with $\beta=v / 2$ for linear failure rate distribution is deduced.

Remark 19. By putting $\alpha=0$ and $\theta=2$ in (20), the recurrence relation for the product moments of gos is deduced for Rayleigh distribution in the form

$$
\begin{aligned}
E\left[X^{i}(r, n, m, k) X^{j}(s, n, m, k)\right]= & \frac{2 \beta \gamma_{s}}{(j+2)}\left\{E\left[X^{i}(r, n, m, k) X^{j+2}(s, n, m, k)\right]\right. \\
& -E\left[X^{i}(r, n, m, k) X^{j+2}(s-1, n, m, k)\right\}
\end{aligned}
$$

\section{Characterizations}

Let $X(r, n, m, k), r=1,2, \ldots, n$ be gos, then the conditional $p d f$ of $X(s, n, m, k)$ given $X(r, n, m, k)=$ $x, 1 \leq r<s \leq n$, in view of (2) and (3), is given by

$$
f_{X(s, n, m, k) \mid X(r, n, m, k)}(y \mid x)=\frac{C_{s-1}}{(s-r-1) ! C_{r-1}}[\bar{F}(x)]^{m-\gamma_{r}+1} \times\left[h_{m}(F(y))-h_{m}(F(x))\right]^{s-r-1}[\bar{F}(y)]^{\gamma_{s}-1} f(y), x<y
$$

Theorem 3. Let $X$ be a non-negative random variable having an absolutely continuous $d f F(x)$ with $F(0)=0$ and $0 \leq F(x) \leq 1$ for all $x>0$, then

$$
E[\xi\{X(s, n, m, k)\} \mid X(l, n, m, k)=x]=e^{-\left(\alpha x+\beta x^{\theta}\right)} \prod_{j=1}^{s-l}\left(\frac{\gamma_{l+j}}{\gamma_{l+j}+1}\right), l=r, r+1
$$

if and only if

$$
\bar{F}(x)=e^{-\left(\alpha x+\beta x^{\theta}\right)}, x>0, \alpha>0, \beta>0, \theta>0
$$

where $\xi(y)=e^{-\left(\alpha y+\beta y^{\theta}\right)}$.

Proof. We have from (23) for $s>r+1$,

$$
\begin{aligned}
E[\xi\{X(s, n, m, k)\} \mid X(r, n, m, k)=x]=\frac{C_{s-1}}{(s-r-1) ! C_{r-1}(m+1)^{s-r-1}} \\
\quad \times \int_{x}^{\infty} e^{-\left(\alpha y+\beta y^{\theta}\right)}\left(\frac{\bar{F}(y)}{\bar{F}(x)}\right)^{\gamma_{s}-1}\left[1-\left(\frac{\bar{F}(y)}{\bar{F}(x)}\right)^{m+1}\right]^{s-r-1} \frac{f(y)}{\bar{F}(x)} \mathrm{d} y
\end{aligned}
$$

By setting $u=\frac{\bar{F}(y)}{\bar{F}(x)}=\frac{e^{-\left(\alpha y+\beta y^{\theta}\right)}}{e^{-\left(\alpha x+\beta x^{\theta}\right)}}$ from (5) in (26), we obtain

$$
E[\xi\{X(s, n, m, k)\} \mid X(r, n, m, k)=x]=\frac{C_{s-1} e^{-\left(\alpha x+\beta x^{\theta}\right)}}{(s-r-1) ! C_{r-1}(m+1)^{s-r-1}} \times \int_{0}^{1} u^{\gamma_{s}}\left(1-u^{m+1}\right)^{s-r-1} \mathrm{~d} u
$$

Again by setting $t=u^{m+1}$ in (27), we get

$$
E[\xi\{X(s, n, m, k)\} \mid X(r, n, m, k)=x]=\frac{C_{s-1} e^{-\left(\alpha x+\beta x^{\theta}\right)}}{(s-r-1) ! C_{r-1}(m+1)^{s-r}} \int_{0}^{1} t^{\frac{\gamma_{s}+1}{m+1}-1}(1-t)^{s-r-1} \mathrm{~d} t
$$

and hence the necessary part given in (24).

To prove the sufficient part, we have from (23) and (24)

$$
\begin{gathered}
\frac{C_{s-1}}{(s-r-1) ! C_{r-1}(m+1)^{s-r-1}} \int_{x}^{\infty} e^{-\left(\alpha y+\beta y^{\theta}\right)}\left[(\bar{F}(x))^{m+1}-(\bar{F}(y))^{m+1}\right]^{s-r-1} \times[\bar{F}(y)]^{\gamma_{s}-1} f(y) \mathrm{d} y \\
=H_{s \mid r}(x)[\bar{F}(x)]^{\gamma_{r+1}}
\end{gathered}
$$

where 


$$
H_{s \mid r}(x)=e^{-\left(\alpha x+\beta x^{\theta}\right)} \prod_{j=1}^{s-r}\left(\frac{\gamma_{r+j}}{\gamma_{r+j}+1}\right)
$$

Differentiating (28) both sides with respect to $x$, we get

$$
\begin{aligned}
& \frac{C_{s-1}[\bar{F}(x)]^{m} f(x)}{(s-r-2) ! C_{r-1}(m+1)^{s-r-2}} \int_{x}^{\infty} e^{-\left(\alpha y+\beta y^{\theta}\right)}\left[(\bar{F}(x))^{m+1}-(\bar{F}(y))^{m+1}\right]^{s-r-2} \\
& \times[\bar{F}(y)]^{\gamma_{s}-1} f(y) \mathrm{d} y=H_{s \mid r}^{\prime}(x)[\bar{F}(x)]^{\gamma_{r+1}}-\gamma_{r+1} H_{s \mid r}(x)[\bar{F}(x)]^{\gamma_{r+1}-1} f(x)
\end{aligned}
$$

or

$$
-\gamma_{r+1} H_{s \mid r+1}(x)[\bar{F}(x)]^{\gamma_{r+2}+m} f(x)=H_{s \mid r}^{\prime}(x)[\bar{F}(x)]^{\gamma_{r+1}}-\gamma_{r+1} H_{s \mid r}(x)[\bar{F}(x)]^{\gamma_{r+1}-1} f(x)
$$

Therefore,

$$
\frac{f(x)}{\bar{F}(x)}=-\frac{H_{s \mid r}^{\prime}(x)}{\gamma_{r+1}\left[H_{s \mid r+1}(x)-H_{s \mid r}(x)\right]}=\alpha+\beta \theta x^{\theta-1}
$$

which proves that

$$
F(x)=1-e^{-\left(\alpha x+\beta x^{\theta}\right)}, x>0, \alpha>0, \beta>0, \theta>0
$$

Remark 20. At $m \rightarrow-1$ in (24), we get the characterization results from the exponential-Weibull distribution based on $k$-th upper record values.

Remark 21. Setting $m=0, k=1$ in (24), we obtain the characterization results of the exponentialWeibull lifetime distribution based on order statistics.

Following theorem contains characterization of this distribution by a recurrence relation for the single moments of gos .

Theorem 4. Fix a positive integer $k$ and let $j$ be a non-negative integer. A necessary and sufficient condition for a random variable $X$ to be distributed with $p d f$ given by (4) is that

$$
\begin{aligned}
E\left(X^{j}(r, n, m, k)\right)= & \frac{\alpha \gamma_{r}}{j+1}\left\{E\left[X^{j+1}(r, n, m, k)\right]-E\left[X^{j+1}(r-1, n, m, k)\right]\right\}+\frac{\beta \theta \gamma_{r}}{j+\theta} \\
& \times\left\{E\left[X^{j+\theta}(r, n, m, k)\right]-E\left[X^{j+\theta}(r-1, n, m, k)\right]\right\}
\end{aligned}
$$

Proof. The necessary part follows immediately from equation (18). On the other hand if the relation in (29) is satisfied, then on using (2), we have

$$
\begin{aligned}
& \frac{C_{r-1}}{(r-1) !} \int_{0}^{\infty} x^{j}[\bar{F}(x)]_{r}^{\gamma_{r}-1} f(x) g_{m}^{r-1}(F(x)) \mathrm{d} x \\
& =\frac{\alpha C_{r-1}}{(r-1) !(j+1)} \int_{0}^{\infty} x^{j+1}[\bar{F}(x)]^{\gamma_{r}} f(x) g_{m}^{r-2}(F(x))\left\{\frac{\gamma_{r} g_{m}(F(x))}{\bar{F}(x)}-(r-1)[\bar{F}(x)]^{m}\right\} \mathrm{d} x \\
& +\frac{\beta \theta C_{r-1}}{(r-1) !(j+\theta)} \int_{0}^{\infty} x^{j+\theta}[\bar{F}(x)]^{\gamma_{r}} f(x) g_{m}^{r-2}(F(x))\left\{\frac{\gamma_{r} g_{m}(F(x))}{\bar{F}(x)}-(r-1)[\bar{F}(x)]^{m}\right\} \mathrm{d} x
\end{aligned}
$$

Let

$$
h(x)=-\left[(\bar{F}(x)]^{\gamma_{r}} g_{m}^{r-1}(F(x))\right.
$$

Differentiating both sides of (30), we get

$$
h^{\prime}(x)=[\bar{F}(x)]^{\gamma_{r}} f(x) g_{m}^{r-2}(F(x))\left\{\frac{\gamma_{r} g_{m}(F(x))}{\bar{F}(x)}-(r-1)[\bar{F}(x)]^{m}\right\}
$$

Thus,

$$
\begin{aligned}
& \frac{C_{r-1}}{(r-1) !} \int_{0}^{\infty} x^{j}[\bar{F}(x)]^{\gamma_{r}-1} f(x) g_{m}^{r-1}(F(x)) \mathrm{d} x \\
& =\frac{\alpha C_{r-1}}{(r-1) !(j+1)} \int_{0}^{\infty} x^{j+1} h^{\prime}(x) d x+\frac{\beta \theta C_{r-1}}{(r-1) !(j+\theta)} \int_{0}^{\infty} x^{j+\theta} h^{\prime}(x) \mathrm{d} x
\end{aligned}
$$

Integrating right hand side in (31) by parts and using the value of $h(x)$ from (30), we find that 


$$
\frac{C_{r-1}}{(r-1) !} \int_{0}^{\infty} x^{j}[\bar{F}(x)]^{\gamma_{r}-1} g_{m}^{r-1}(F(x))\left\{\left(\alpha+\beta \theta x^{\theta-1}\right) \bar{F}(x)-f(x)\right\} \mathrm{d} x=0
$$

Applying the extension of Müntz-Szász Theorem, (see for example Hwang and Lin [28]) to (32), we get

$$
f(x)=\left(\alpha+\beta \theta x^{\theta-1}\right) \bar{F}(x)
$$

which proves that $f(x)$ has the form as in (6).

Remark 22. Theorem 4 can be used to characterize the exponential, Weibull, linear failure rate and Rayleigh distributions by setting $\beta=0, \alpha=0, \theta=2$ and $\alpha=0, \theta=2$, respectively.

Acknowledgments. The authors acknowledge with thanks to the referees and the Editor-in-Chief for their fruitful suggestions and comments which led to the overall improvement in the manuscript.

\section{References}

1. U. Kamps, A Concept of Generalized Order Statistics, B.G. Teubner Stuttgart, Germany, 1995.

2. U. Kamps and U. Gather, "Characteristic property of generalized order statistics for exponential distribution," Applicationes Mathematicae (Warsaw), vol. 24, no. 4, pp. 383-391, 1997.

3. C. Keseling, "Conditional distributions of generalized order statistics and some characterizations," Metrika, vol. 49, no. 1, pp. 27-40, 1999.

4. E. Cramer, and U. Kamps, "Relations for expectations of functions of generalized order statistics," Journal of Statistical Planning and Inference, vol. 89, no. 1-2, pp. 79-89, 2000.

5. M. Ahsanullah, "Generalized order statistics from exponential distribution," Journal of Statistical Planning and Inference, vol. 85, no. 1-2, pp. 85-91, 2000.

6. M. Habibullah and M. Ahsanullah, "Estimation of parameters of a Pareto distribution by generalized order statistics," Communications in Statistics - Theory and Methods, vol. 29, no.7, pp. 1597-1609, 2000.

7. M.Z. Raqab, "Optimal prediction-intervals for the exponential distribution based on generalized order statistics," IEEE Transactions on Reliability, vol. 50, no. 1, pp. 112-115, 2001.

8. U. Kamps and E. Cramer, "On distributions of generalized order statistics," Statistics, vol. 35, no. 3, pp. 269$280,2001$.

9. A.A. Ahmad and A.M. Fawzy, "Recurrence relations for single moments of generalized order statistics from doubly truncated distribution," Journal of Statistical Planning and Inference, vol. 117, no. 2, pp. 241-249, 2003.

10. M. Bieniek and D. Szynal, "Characterizations of distributions via linearity of regression of generalized order statistics," Metrika, vol. 58, no. 3, pp. 259-271, 2003.

11. E.K. Al-Hussaini and A.A. Ahmad, "On Bayesian predictive distributions of generalized order statistics," Metrika, vol. 57, no. 2, pp. 165-176, 2003

12. E. Cramer, U. Kamps and C. Keseling, "Characterization via linear regression of ordered random variables: a unifying approach," Communications in Statistics - Theory and Methods, vol. 33, no. 12, pp. 2885-2911, 2004.

13. A.H. Khan and A.A. Alzaid, "Characterization of distributions through linear regression of non-adjacent generalized order statistics," Journal of Applied Statistical Science, vol. 13, pp. 123-136, 2004.

14. Z.F. Jaheen, "Estimation based on generalized order statistics from the Burr model," Communications in Statistics-Theory and Methods, vol. 34, no. 4, pp. 785-794, 2005.

15. A.H. Khan, R.U. Khan and M. Yaqub, "Characterization of continuous distributions through conditional expectation of function of generalized order statistics," Journal of Applied Probability 83 Statistics, vol. 1, no. 1, pp. 115-131, 2006.

16. R.U. Khan, D. Kumar and H. Athar, "Moments of generalized order statistics from Erlang-truncated exponential distribution and its characterization," International Journal of Statistics and System, vol. 5, no. 4, pp. 455-464, 2010.

17. R.U. Khan and B. Zia, "Generalized order statistics from doubly truncated linear exponential distribution and a characterization," Journal of Applied Probability \& Statistics, vol. 9, no. 1, pp. 53-65, 2014.

18. U. Kamps, Characterizations of distributions by recurrence relations and identities for moments of order statistics. In: N. Balakrishnan, N. and C.R. Rao. Handbook of Statistics 16, Order Statistics: Theory \& Methods, North-Holland, Amsterdam, 1998. 
19. G.M. Cordeiro, E.M.M. Ortega, and A.J. Lemonte, "The exponential-Weibull lifetime distribution," Communications in Statistics - Simulation and Computation, vol. 84, no. 12, pp. 2592-2606, 2014.

20. I.S. Gradshteyn and I.M. Ryzhik, Table of Integrals, Series and Products, 7th edition, Academic Press, New York, 2007.

21. S.M. Ruiz, "An algebraic identity leading to Wilson's theorem," The Mathematical Gazette, vol. 80, no. 489, pp. 579-582, 1996.

22. H.A. David and H.N. Nagaraja, Order Statistics, 3rd edition, John Wiley, New York, 2003.

23. H. Athar and H.M. Islam, "Recurrence relations for single and product moments of generalized order statistics from a general class of distribution," Metron, vol. LXII, no. 3, pp. 327-337, 2004.

24. R.U. Khan, A. Kulshreshtha and M.A. Khan, "Relations for moments of k-th record values from exponentialWeibull lifetime distribution," Journal of the Egyptian Mathematical Society, vol. 23, no. 3, pp. 558-562, 2015.

25. R.U. Khan, Z. Anwar and H. Athar, "Recurrence relations for single and product moments of generalized order statistics from doubly truncated Weibull distribution," The Aligarh Journal of Statistics, vol. 27, pp. 69-79, 2007.

26. P. Pawlas and D. Szynal, "Recurrence relations for single and product moments of generalized order statistics from Pareto, generalized Pareto, and Burr distributions," Communications in Statistics - Theory and Methods, vol. 30, no. 4, pp. 739-746, 2001.

27. A.A. Ahmad, "Single and product moments of generalized order statistics from linear exponential distribution," Communications in Statistics - Theory and Methods, vol. 37, no. 8, pp. 1162-1172, 2008.

28. J.S. Hwang and G.D. Lin, "On a generalized moments problem II," Proceedings of the American Mathematical Society, vol. 91, no. 4, pp. 577-580, 1984. 